\title{
Semi-Automated Analysis of Doppler Images for Quantification of Changes in Mitral Inflow Pattern during Parabolic Flight
}

\author{
EG Caiani ${ }^{1}$, G Asquer ${ }^{1}$, E Meraviglia ${ }^{1}$, S Cerutti $^{1}$, M Turiel $^{2}$, \\ O Bailliart ${ }^{3}$, B Cholley ${ }^{3}$, A Capderou ${ }^{4}$, P Vaida $^{5}$ \\ ${ }^{1}$ Dipartimento di Bioingegneria, Politecnico di Milano, Milano, Italy \\ ${ }^{2}$ Cardiology Unit, Istituto Ortopedico Galeazzi, Università degli Studi di Milano, Milano, Italy \\ ${ }^{3}$ CHU Lariboisière, Paris, France \\ ${ }^{4}$ Université Paris Sud UPRES EA 2397, Le Plessis Robinson, France \\ ${ }^{5}$ Université Victor Segalen Bordeaux 2 and CHU Bordeaux, Médecine Aérospatiale, France
}

\begin{abstract}
To study changes in left ventricular (LV) mitral inflow induced by gravitational stresses, and evaluate the effects due to different postures, we performed transthoracic Doppler imaging during parabolic flights on 7 normal subjects (mean age $\square S D, 46 \pm 5$ years) in standing upright and resting position. To detect Doppler velocity profiles, a semi-automatically procedure based on local image properties, was applied. Then, LV inflow parameters were automatically computed and their modifications according to different gravity conditions were found: in standing, $L V$ diastolic rapid filling parameters were significantly affected (E-wave peak, cm/s: from $54.7 \pm 11.1$ at $1 G$ to $85.8 \pm 12.1$ at $0 G ;$ E-wave integral, cm: from $6.6 \pm 1.7$ at $1 G$ to $13.1 \pm 3.2$ at $0 G ;$ E/A integral ratio, a.u.: from $1.4 \pm 7$ at $1 G$ to $2.5 \pm 8$ at $0 G$ ), while late diastolic filling parameters were preserved (A-wave peak, $\mathrm{cm} / \mathrm{s}$ : from $48.8 \pm 3.6$ at $1 G$ to $55.3 \pm 11.3$ at $0 G$ ). No changes were noted while subjects were in supine resting.
\end{abstract}

\section{Introduction}

Modifications in gravity level (head-to-foot acceleration, $\mathrm{Gz}$ ) cause shifts in fluid from the lower extremities toward the head and thorax, altering central filling volumes and pressures, resulting in significant cardiovascular effects [1-2]. The evaluation of these changes, induced by parabolic flight, is important for the definition and testing of appropriate countermeasures to balance the effects of weightlessness on the cardiovascular system to prevent post-flight orthostatic intolerance in astronauts [3], as well as for the better understanding of the human physiology. Transthoracic Doppler echocardiography is an imaging technique routinely used in clinical settings for the evaluation of transvalvular flow velocity patterns. Because of its portability it has been utilized both during space and parabolic flights to study long-term and short-term modifications in cardiac preload or afterload due to microgravity [4-5]. The present study involves Doppler data, as a part of a more complete 2D, 3D and Doppler database, acquired during a particular experimental protocol performed by parabolic flight aimed at evaluating the hemodynamic effects on the cardiovascular system due to abrupt changes in gravity. As concerns this sub-protocol, our primary goal was to focus on the mitral Doppler inflow velocity pattern, reflecting the diastolic properties of the left ventricle (LV), and study its modifications during the parabola, with the subjects in different postures. Usually, the clinical interpretation of Doppler images is based on manual tracing, thus being time-expensive and introducing subjectivity in the analysis. Accordingly, our aim was to develop a procedure, based on local image properties, to semiautomatically detect the enveloped contour of the mitral Doppler inflow profile and automatically extract the conventional clinical parameters useful to characterize the diastolic properties of the LV.

\section{Methods}

\subsection{Experimental protocol}

Parabolic flights were performed in the Zero-G Airbus A300, managed by Novespace for CNES and ESA in Bordeaux, France. A flight campaign consists in three days of flight, each lasting about 3 hours and consisting of 31 parabolas. Each parabola included four consecutive phases: normogravity $(1 \mathrm{Gz})$, before the parabola begun; mild hypergravity $(1.8 \mathrm{Gz})$ during the ascending phase of the parabola, microgravity $(0 \mathrm{Gz})$ lasting for about $20 \mathrm{~s}$; a second period of mild hypergravity $(1.8 \mathrm{Gz})$ during the descending phase of the parabola (20-25 sec), followed by a new steady state at $1 \mathrm{Gz}$ (about $2 \mathrm{~min}$ ). 

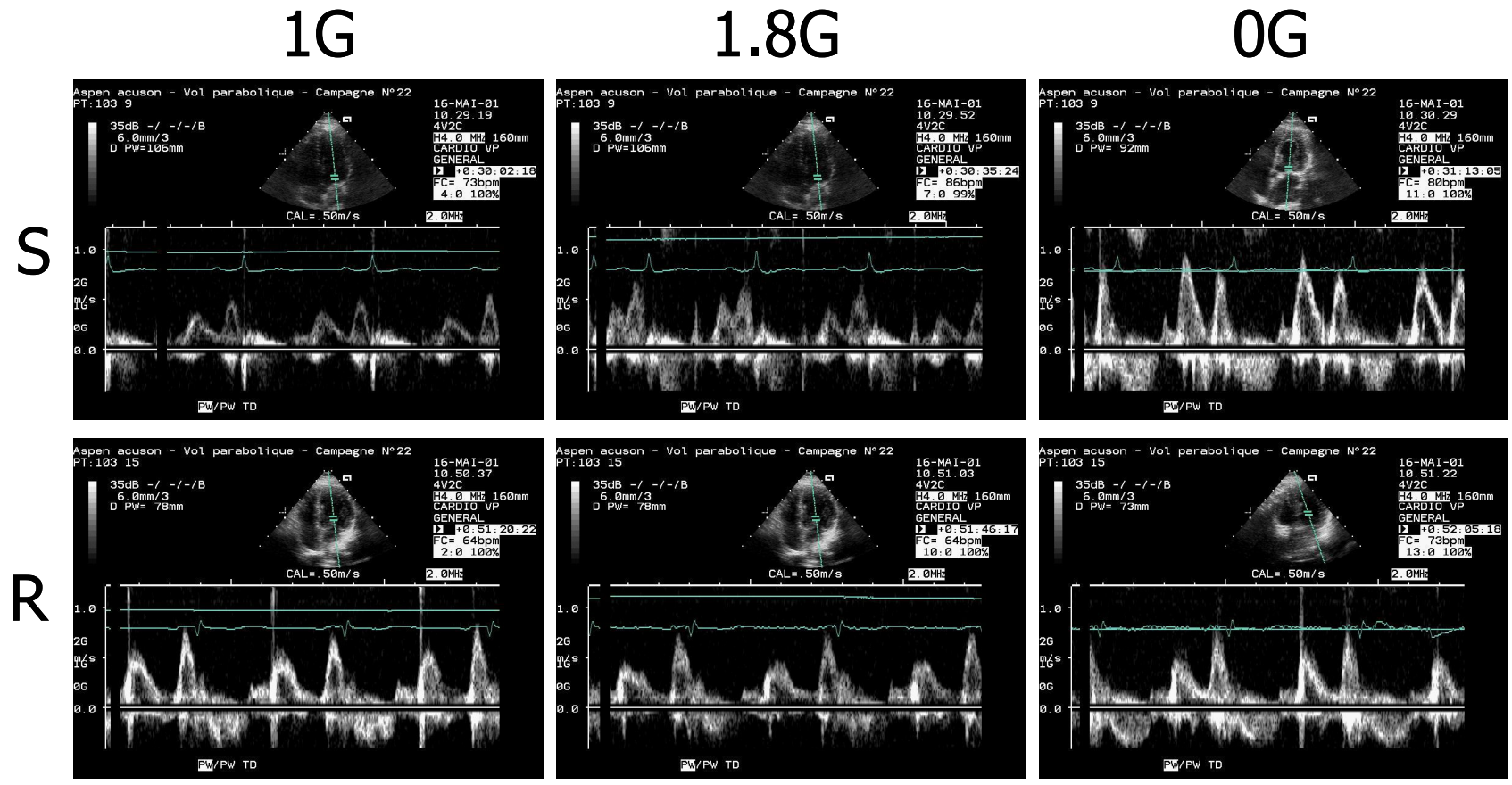

Figure 1. Examples of LV mitral inflow Doppler images acquired in the same subjects during different gravity phases (1G: normogravity, 1.8G: hypergravity, 0G: microgravity) in the parabola (from left to right), in standing upright (S, top panels) and supine resting ( $\mathrm{R}$, bottom panels) positions.

A group of seven healthy and unmedicated subjects (mean age $\pm \mathrm{SD}, 46$ age \pm 5 years) was studied in standing upright position and in supine resting, in different parabolas respectively. After careful positioning, utilizing a conventional apical 4-chamber view (Aspen, Acuson, Siemens, France), of the sample volume inside the LV cavity, pulsed-Doppler modality (4 $\mathrm{MHz}$ transducer) was activated and images relevant to the LV mitral inflow velocity profile (Figure 1) were continuously acquired as series of still frames on a magneto-optical disk, with care to maintain the sample volume location stable during the parabola.

\subsection{Data analysis}

In each subject, for each experimental condition (standing and resting), at each gravity phase $(1 \mathrm{Gz}$, first $1.8 \mathrm{Gz}$ and $0 \mathrm{Gz}$ ) three consecutive beats corresponding to good quality data were selected for the analysis.

Images were processed as follows by specifically designed custom software. First, the region of interest containing the Doppler signal was automatically cropped and the ECG signal and the gravity signals removed from the image. Then, a horizontal Sobel filter was utilized to detect the baseline and thus determine the positive velocity subimage. To semi-automatically detect the Doppler flow velocity profile, an algorithm based on histogram thresholding was applied. A scan for each image column was performed from the top to the bottom, to search for the first pixel with videointensity greater than a predefined threshold $\mathrm{T}$, which was assumed as part of the contour of the velocity profile.

For each image separately, to automatically define the value of $\mathrm{T}$, the image histogram was calculated. Then, the value of the threshold $\mathrm{T}$ was assumed as the pixel intensity value such as the $25 \%$ of pixels in the image resulted over the threshold, where the value of $25 \%$ was experimentally obtained as the best choice. From the obtained binary image created by the thresholding operation, the velocity signal was extracted and filtered with a median filter, to remove outliers, and then overimposed to the original image (Figure 2) to allow visual verification and manual change of the threshold value, if necessary.

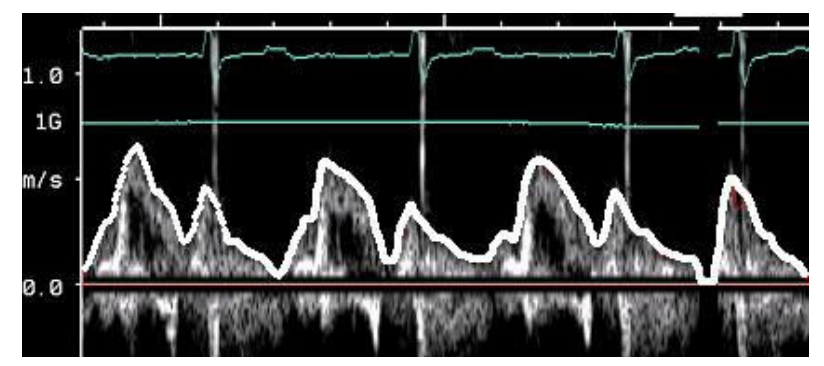

Figure 2. Example of the semi-automatically detected velocity profile overimposed to the original Doppler image. 


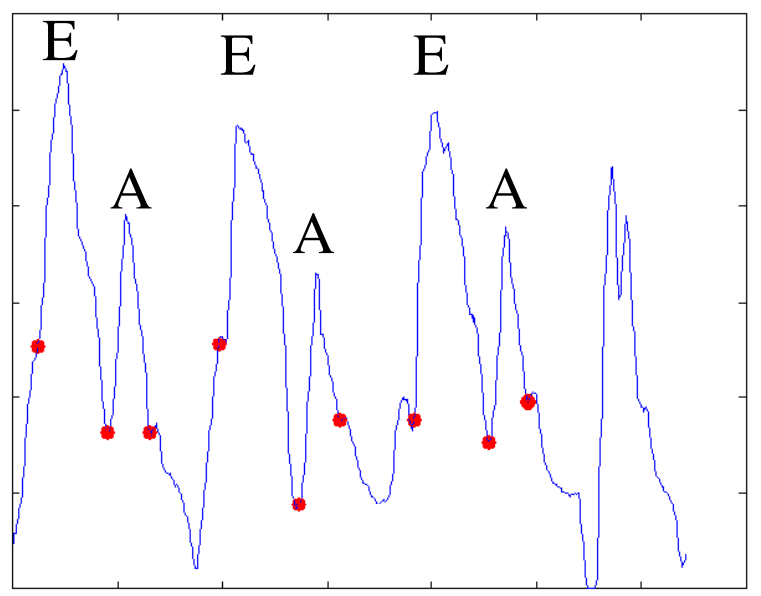

Figure 3. Extracted Doppler velocity profile with the automatically detected $\mathrm{E}$ and $\mathrm{A}$ wave starting and ending points.

The $\mathrm{E}$ and $\mathrm{A}$ wave peak velocity fiduciary points were then detected as relative maximums present in the velocity profile, and utilized for the identification in the profile of the different whole cardiac beats. Then, the starting and ending points of the $\mathrm{E}$ and $\mathrm{A}$ wave were automatically detected (Figure 3) and the following LV inflow parameters computed for each beat: peak, mean and integral of $\mathrm{E}$ and $\mathrm{A}$ velocity waves, deceleration time, E/A peak velocity and integral ratios. The results obtained from three consecutive beats were then averaged to reduce measure variability and take into account changes due to respiration.

\subsection{Statistical analysis}

One way analysis of variance (F-test, Tukey test, $\mathrm{p}<0.05$ ) was performed to test differences in Doppler parameters among the different gravity phases, for each posture condition separately.

\section{Results}

A total of 42 frames was analyzed, with a mean computation time of 3 minutes/frame, using a Pentium III $1300 \mathrm{MHz}$ personal computer.

The proposed method was able to reliably detect the Doppler velocity contour, as assessed by visual inspection, in all the analyzed frames, with minimal need of user interaction for threshold adjustment. An example of the detected contours superimposed to the original images is shown in Figure 2.

In table I, the mean values of the computed parameters of LV mitral inflow are reported, for each experimental condition separately (standing and resting), relevant to each gravity phase $(1 \mathrm{G}, 1.8 \mathrm{G}, 0 \mathrm{G})$.
In subjects entering microgravity in the standing position, significant changes in $\mathrm{E}$ wave (peak velocity, mean velocity, velocity integral) were observed. In particular, E wave peak rose $57 \%$ between $1 \mathrm{Gz}$ and $0 \mathrm{Gz}$, mean early LV filling velocity increased of $54 \%$, while $\mathrm{E}$ wave integral was doubled.

The LV inflow relevant to atrial contraction (A wave) was not significantly increased during microgravity, resulting in an increase of the E/A ratio indices of $79 \%$ (between integrals) and $39 \%$ (between peaks). Deceleration time showed a trend of increase at $0 \mathrm{Gz}$, even not significant. No significant differences in LV inflow parameters were noticed while subjects were under hypergravity $(1.8 \mathrm{G})$ conditions.

When subjects were in the supine resting position, no changes were found in both early and late LV inflow parameters among different gravity phases.

\section{Discussion and conclusions}

This study utilized Doppler echocardiography to noninvasively investigate the LV filling properties during the different gravity phases induced by parabolic flight.

Parabolic flight offers a unique experimental condition to study acute non pharmacologically induced variations of LV mitral inflow, which are reversible and repeatable in the same subject during the flight.

The automated procedure implemented for objective extraction of Doppler parameters allowed us to reduce the subjectivity of the results, avoiding manual tracing. Despite the not optimal quality of the images, due to the experimental conditions, the proposed algorithm was able to reliably detect the Doppler contour.

Expected changes in LV inflow parameters associated with different gravity conditions were observed. In particular, in standing position, microgravity significantly and acutely affected LV diastolic rapid filling, while late diastolic filling appeared preserved. This could be related to the increase in venous return, due to changes in hydrostatic pressure associated with $0 \mathrm{G}$ which results in left atrial dilatation and atrial pressure overload. This increase in left atrial pressure generates an high atrioventricular pressure gradient, which results in an increased $\mathrm{E}$ wave.

On the contrary, while the subjects were in the supine resting position, there was no or minimal gradient in hydrostatic pressure between head, heart and legs, thus minimizing the blood redistribution when microgravity phase was achieved. This posture led to no changes in LV mitral inflow parameters.

This findings could give more insight to our previous results obtained by studying changes in LV dimensions during parabolic flight [6]: the LV dilatation observed during microgravity could be interpreted as due to an increase in diastolic rapid filling, while contribution of 
Table 1. Results (mean \pm SD) of the LV Doppler mitral inflow parameters obtained during the parabola in standing and in resting supine position (*: $\mathrm{p}<0.05$ versus $1 \mathrm{Gz}$ ) .

\begin{tabular}{l|ccc|ccc|} 
& \multicolumn{3}{|c|}{ STAND } & \multicolumn{3}{c|}{ REST } \\
& $1 \mathrm{Gz}$ & $1.8 \mathrm{Gz}$ & $0 \mathrm{Gz}$ & $1 \mathrm{Gz}$ & $1.8 \mathrm{Gz}$ & $0 \mathrm{Gz}$ \\
\hline Peak E wave (cm/s) & $54.7 \pm 11.1$ & $53.2 \pm 9.6$ & $85.8 \pm 12.1^{*}$ & $65.0 \pm 16.4$ & $63.0 \pm 15.1$ & $66.0 \pm 15.0$ \\
Mean E wave velocity (cm/s) & $30.0 \pm 6.0$ & $30.1 \pm 5.1$ & $46.2 \pm 7.2^{*}$ & $34.1 \pm 8.2$ & $33.8 \pm 9.0$ & $35.3 \pm 8.0$ \\
E wave integral (cm) & $6.6 \pm 1.7$ & $6.0 \pm 2.6$ & $13.1 \pm 3.2^{*}$ & $10.4 \pm 3.1$ & $10.1 \pm 2.4$ & $9.5 \pm 2.5$ \\
Peak A wave (cm/s) & $48.8 \pm 3.6$ & $58.0 \pm 7.9$ & $55.3 \pm 11.3$ & $54.6 \pm 9.0$ & $52.6 \pm 11.2$ & $54.4 \pm 11.2$ \\
Mean A wave velocity (cm/s) & $27.3 \pm 4.0$ & $32.3 \pm 4.4$ & $31.7 \pm 9.0$ & $28.6 \pm 4.1$ & $28.0 \pm 5.9$ & $29.0 \pm 6.1$ \\
A wave integral (cm) & $5.5 \pm 1.5$ & $7.5 \pm 2.3 *$ & $5.7 \pm 1.4$ & $6.6 \pm 0.9$ & $5.5 \pm 1.0$ & $5.9 \pm 0.9$ \\
Deceleration time (ms) & $160 \pm 40$ & $141 \pm 68$ & $209 \pm 47$ & $166 \pm 69$ & $157 \pm 58$ & $124 \pm 46$ \\
E/A integral (a.u.) & $1.40 \pm 0.73$ & $1.08 \pm 0.76$ & $2.51 \pm 0.80^{*}$ & $1.62 \pm 0.55$ & $1.91 \pm 0.5$ & $1.62 \pm 0.41$ \\
E/A peak (a.u.) & $1.14 \pm 0.23$ & $0.94 \pm 0.21$ & $1.59 \pm 0.25^{*}$ & $1.23 \pm 0.37$ & $1.26 \pm 0.4$ & $1.25 \pm 0.24$ \\
Cardiac frequency (bpm) & $78 \pm 6$ & $86 \pm 6$ & $74 \pm 11$ & $69 \pm 8$ & $70 \pm 10$ & $74 \pm 5$ \\
\hline
\end{tabular}

atrial contraction appeared not determinant in further increasing the LV volume.

In previous studies [7], Johns et co-authors found that $\mathrm{LV}$ filling was more prominently enhanced when $0 \mathrm{Gz}$ was entered while subjects were supine, in respect to what they found in sitting subjects, suggesting a shift of fluids within the pulmonary vasculature. Discrepancies with our results could be attributed to: 1) the 'roller coaster' parabolic flight profile they utilized, with only few seconds between consecutive parabolas, that does not allow to return to normal conditions between consecutive parabolas; 2) the younger population (mean age 31 years old) they studied.

Our results confirmed the fact that LV mitral inflow is greatly affected by changes in subject's loading conditions, and mitral inflow profile can reflect these changes in a few seconds. This has to be taken into account in clinical settings when Doppler evaluation is usually performed without a direct measure of the patient's loading condition.

\section{Acknowledgements}

We are grateful to Pierre Téchoueyres for his technical support. A special thank to the Microgravity Division of CNES, ESA, and Novespace for the organization of the parabolic flights campaign, and in particular to Christophe Mora and Fréderic Gai from Novespace. Pierre Vaïda was the recipient of grants 02/CNES/480000039 and CNES/2003/4800000162.

\section{References}

[1] Lathers CM, Charles JB, Elton KF, Holt TA, Mukai C, Bennet BS, Bungo MW. Acute hemodynamic responses to weightlessness in humans. J Clin Pharmacol 1989;29:61527

[2] Norsk P, Foldager N, Bonde-Petersen F, Elmann-Larsen B, Johansen TS. Central venous pressure in humans during short periods of weightlessness. J Appl Physiol 1987;63:2433-2437

[3] Guell A, Braak L. Cardiovascular deconditioning syndrome during space flight. Ann Cardiol Angiol 1989;38(8):499-502

[4] Martin DS, South DA, Garcia KM, Arbeille P. Ultrasound in space. Ultrasound Med. Biol 2003:29(1):1-12

[5] Mukai CN, Lathers C.M, Charles JB, Bennet BS. Cardiovascular responses to repetitive exposure to hyperand hypogravity states produced by parabolic flights. J Clin Pharmacol 1994;34:472-9

[6] Corsi C, Lamberti C, Cerutti S, Laulom JP, Bailliart O, Cholley B, Capderou A, Vaida P, Caiani EG. Level set based technique for the quantification of left ventricular size and function in 2-D echocardiography during parabolic flights. Med \& Biol Eng \& Comp 2004;42 (in press)

[7] Johns JP, Vernalis MN, Karemaker JM, Latham RD. Doppler evaluation of cardiac filling and ejection properties in humans during parabolic flight. J Appl Physiol 1994;76:2621-2626.

Address for correspondence

Enrico G Caiani

Dipartimento di Bioingegneria, Politecnico di Milano

Piazza L. da Vinci, 32

20133 Milano, ITALY

enrico.caiani@biomed.polimi.it 